\title{
Hypertrophic cardiomyopathy associated with a mitochondrial myopathy of voluntary muscles and congenital cataract
}

\author{
R C A SENGERS, * A M STADHOUDERS, $†$ E VAN LAKWIJK-VONDROVICOVA, \\ K KUBAT,§W RUITENBEEK*
}

From the Departments of *Paediatrics, $\dagger$ Submicroscopic Morphology, $\ddagger$ Cardiology, and §Pathology, University of Nijmegen, Nijmegen, The Netherlands

SUMMARY Structurally abnormal mitochondria were found in skeletal muscle cells from a woman with hypertrophic cardiomyopathy and myopathy of voluntary muscles associated with congenital cataracts. Moderate exercise resulted in lactic acidosis. Oxidation of pyruvate and other substrates and the production of adenosine triphosphate were normal in vitro. A younger brother of the patient had had congenital cataract and had died from hypertrophic obstructive cardiomyopathy.

The concurrent occurrence of pathological changes in both heart and skeletal muscle is a feature of several inherited diseases such as Duchenne and Becker muscular dystrophies. The heart and voluntary muscles are also both affected in several diseases with a well defined enzyme deficiency, such as Pompe's disease, and in other diseases in which the molecular defect is unknown.

Ten years ago we described seven patients from three unrelated families who had hypertrophic cardiomyopathy and a mitochondrial myopathy of skeletal muscle. ${ }^{1}$ They had associated symptoms of congenital cataracts and exercise related lactic acidosis. The syndrome is now registered as an inherited disease 21235 on the McKusick register. ${ }^{2}$ Recently we had the opportunity to study two other patients from one family who had this syndrome. On this occasion we are able to perform biochemical studies on skeletal muscle tissue from one of the patients.

\section{Case reports}

PATIENT 1

This woman of 33 was the third of five children of healthy non-consanguineous parents. Within a few weeks of birth, bilateral cataracts were identified. These were surgically removed when she was 6 months old. She always became very tired during

Requests for reprints to Dr RCA Sengers, Department of Paediatrics, University of Nijmegen, PO Box $9101,6500 \mathrm{HB}$ Nijmegen, The Netherlands.

Accepted for publication 12 July 1985 exercise and she could never compete physically with her peers. At the age of 25 years she was first admitted to our hospital for cardiological examination because her brother had died from a cardiomyopathy. Her other sibs were healthy. She was known to have had a heart murmur for about 20 years. Her exercise tolerance worsened with activity, but did not change consistently with time of day, food intake, or menstrual cycle. She denied progression of her exercise intolerance, and also had no dysarthria, dysphagia, diplopia, muscle cramps, fasciculations, or myotonia. She did not drink alcohol to excess or take drugs, other than the occasional aspirin. General examination at the age of 33 years was unremarkable except for moderate obesity (height $160 \mathrm{~cm}$, weight $50 \mathrm{~kg}$ ). She was visually handicapped by aphakia. Her mental state, cranial nerve function, muscle bulk and tone, tendon reflexes, sensation, and coordination were normal. Muscle strength during individual movements was normal but her endurance was extremely limited. Cardiological examination showed cardiomegaly and a grade II systolic murmur at the left sternal border. Blood pressure was $120 / 70 \mathrm{~mm} \mathrm{Hg}$. There was electrocardiographic evidence of hypertrophy of the right and left ventricles (Fig. 1) and apical hypertrophic cardiomyopathy was diagnosed by echocardiography. The interventricular septum measured $16 \mathrm{~mm}$ and the posterior left ventricular wall $13 \mathrm{~mm}$ at the level of the mitral valve. The wall and septum were both $18 \mathrm{~mm}$ thick at the apex. Doppler studies did not show flow velocity abnormalities. During exercise testing on a bicycle ergometer she 


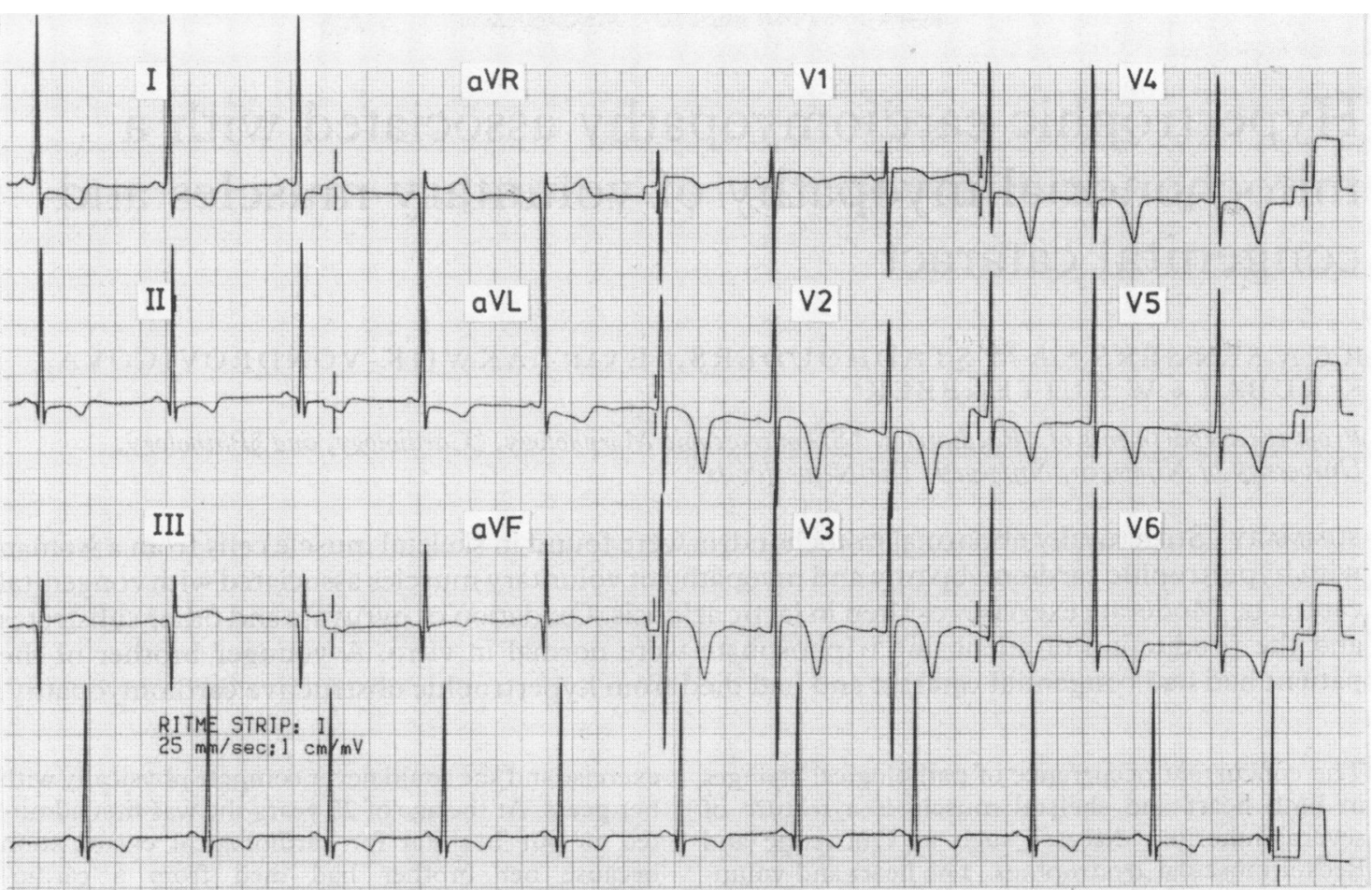

Fig. 1 Electrocardiogram in patient 1 showing evidence of hypertrophy of the right and left ventricles.

was not able to perform at the $40 \mathrm{~W}$ level for more than a few minutes before she became exhausted. Laboratory examination showed a normal arterial $\mathrm{pH}$ and lactic acid concentration at rest but a serious metabolic acidosis $(\mathrm{pH}, 7.28$; bicarbonate 15.4 $\mathrm{mmol} / \mathrm{l}$; base excess, $-9.9 \mathrm{mmol} / \mathrm{l})$ and raised lactic acid concentration $(10.4 \mathrm{mmol} / \mathrm{l}$, normal $<1.8$ $\mathrm{mmol} / \mathrm{l}$ ) after submaximal exercise. Lactic acid concentration after 5 minutes' exercise on a treadmill at a speed of 2 miles per hour ( $3.2 \mathrm{~km}$ per hour) and an inclination of $10 \%$ increased by $3.6 \mathrm{mmol} / 1$ and $\mathrm{pH}$ fell from $7 \cdot 37$ to $7 \cdot 22$. (There were no changes in controls). Serum creatine kinase concentration was normal. Maximum heart rate was 140 beats per min. A specimen of the patient's quadriceps muscle was obtained by open biopsy after she had given informed consent.

\section{PATIENT 2}

A younger brother of patient 1 was admitted to our clinic at the age of 18 years with cardiomegaly. At the age of 5 years he also had had congenital cataracts removed. He suffered from exercise intolerance. General examination revealed no severe abnormalities. Blood pressure was $135 / 80 \mathrm{~mm} \mathrm{Hg}$. A chest $x$ ray examination showed cardiomegaly and a grade II systolic murmur was heard at the left sternal border and in the second right intercostal space. Hypertrophy of the left ventricle and of the interventricular septum was diagnosed by electrocardiography and vector cardiography. Heart catheterisation showed normal pressures. The tip of the catheter, however, could not be placed in the most distal part of the left ventricle. The cineangiographic findings were typical of hypertrophic obstructive cardiomyopathy. Subjectively he remained well until he was 23 years old when he was readmitted with an attack of cardiac asthma. He responded well to conventional treatment. Shortly afterwards, however, cardiac asthma developed again and he died. The most important finding at necropsy was myocardial hypertrophy. The heart weighed $1220 \mathrm{~g}$. Hypertrophy of the septum and the left ventricle had seriously narrowed the outflow tract. The right ventricle was also hypertrophied. The heart valves were intact and there were no defects in the atrial or ventricular septa. The coronary arteries were normal.

\section{HISTOPATHOLOGICAL EXAMINATION \\ Skeletal muscle}

Muscle fibres from patient 1 did not vary greatly in size or shape. No necrotic fibres were seen and there was no inflammation. Connective tissue, nerve bundles, and blood vessels were normal. Haematoxylin 

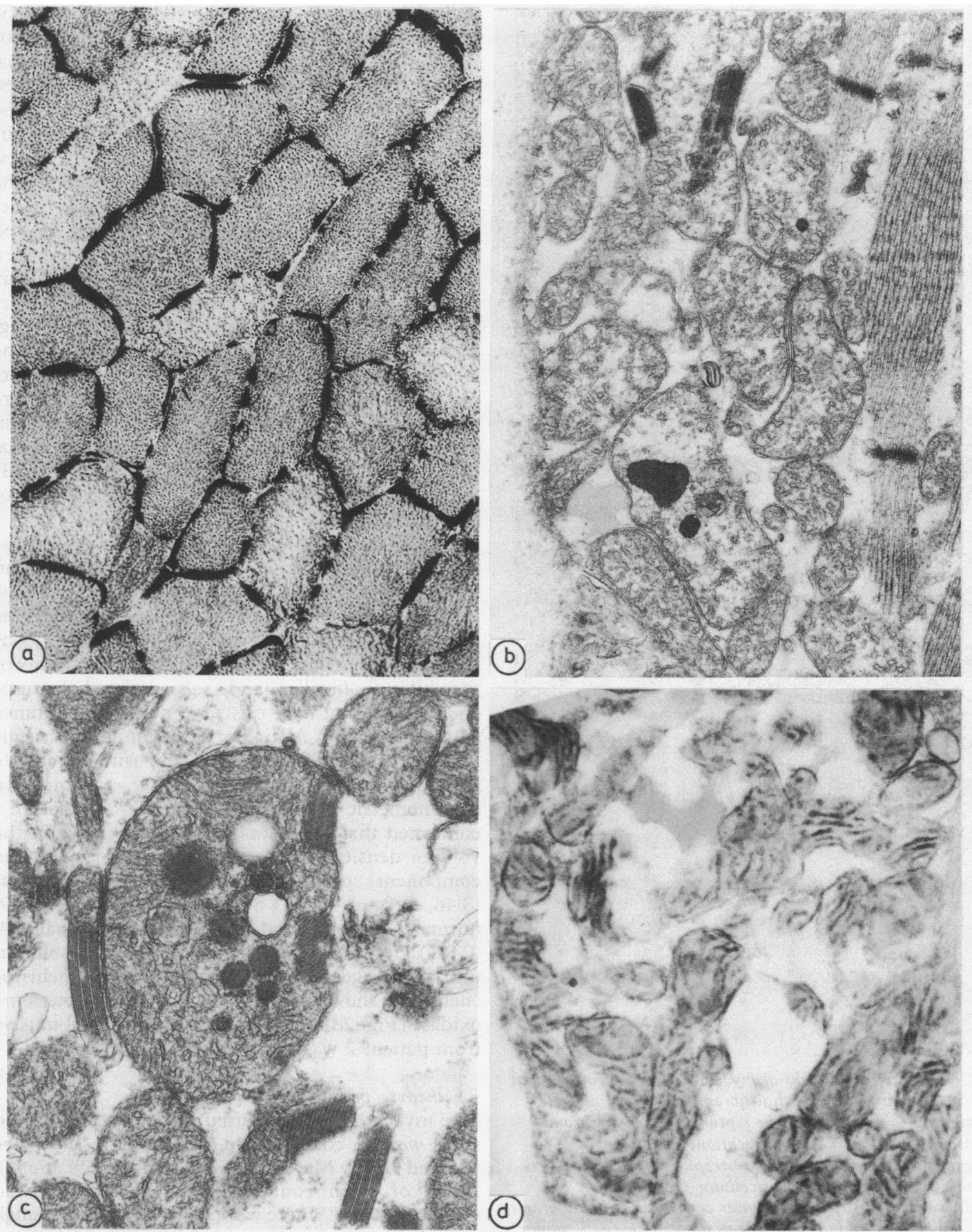

Fig. 2 Various mitochondrial abnormalities seen in a biopsy specimen of the quadriceps muscle from patient 1.

(a) Low power photomicrograph of a transversely sectioned muscle fibre stained by means of the succinic dehydrogenase reaction to show the mitochondria. Note the strong staining of the subsarcolemmas of most muscle fibres. No ragged red fibres were seen. Original magnification, $\times 270$. (b) Low power electron micrograph of subsarcolemmal area showing poorly developed cristae in mitochondria and electron dense matrix deposits. Original magnification, $\times 22000$. (c) High power electron micrograph of giant mitochondrion with crystalline and amorphous inclusions. Original magnification, $\times 93000$. (d) Electronmicrograph showing histochemical reaction to cytochrome oxidase by aberrant subsarcolemmal mitochondria. Original magnification, $\times 18000$. 


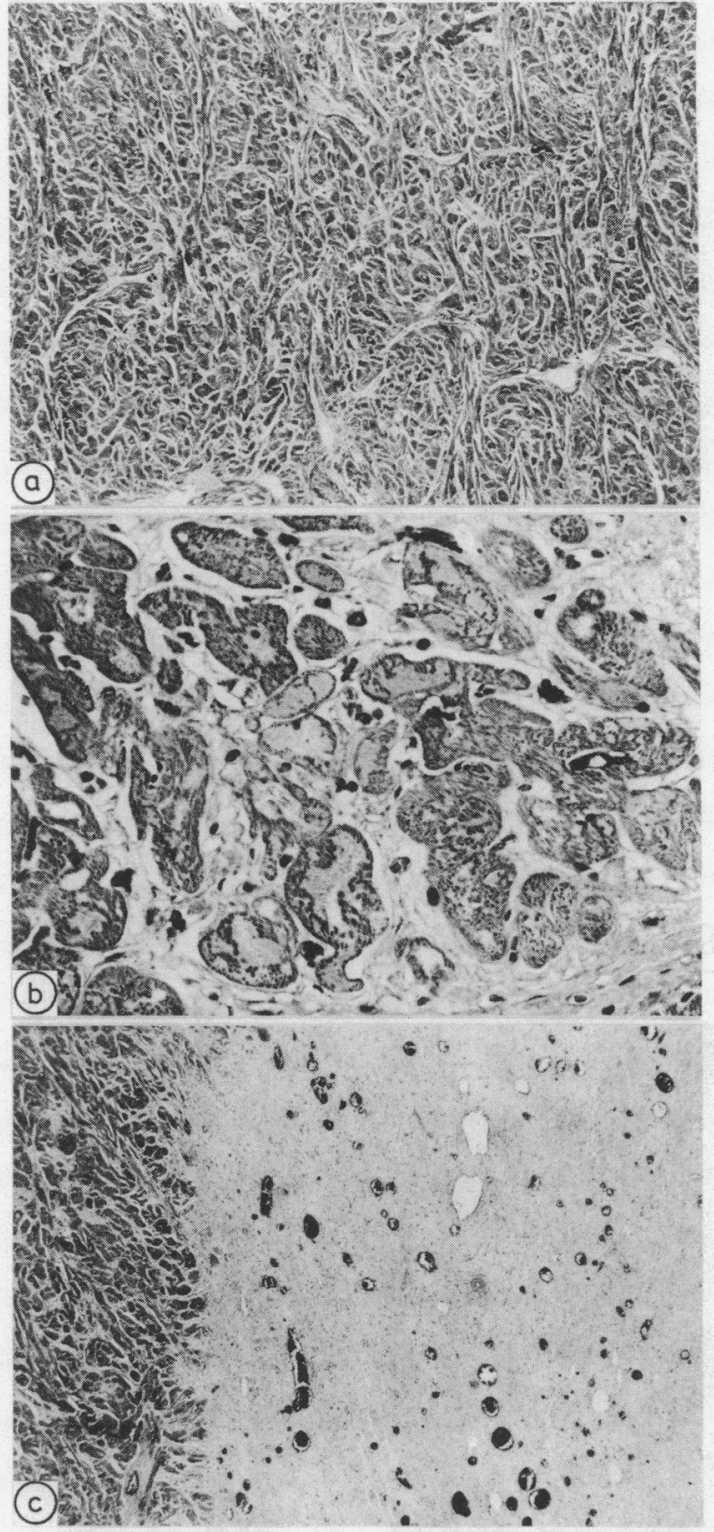

Fig. 3 Photomicrographs of myocardial fibres from patient 2. (a) Disarrayed myocardial fibres of type IIA and IIB (according to Maron ${ }^{4}$ ) in the septum. Elastin trichrome Masson stain. Original magnification, $\times 35$. (b) Myofibrillolysis and vacuolar dystrophy of severely hypertrophic muscle fibres with pericellular fibrosis. These are small scars in left upper and lower corner. Elastin trichrome Masson stain. Original magnification, $\times 350$. (c) Laminar scar seen in the middle third of the ventricular septum. Elastin trichrome Masson stain. Original magnification, $\times 35$. and eosin stained sections showed that most of the muscle fibres contained an increased amount of bluish red granular material in the subsarcolemmal regions. In preparations stained by the modified Gomori trichrome technique red granular material was seen beneath the sarcolemma in numerous fibres, but there were no distinct "ragged red" fibres.

The number of lipid droplets between the myofibrils and under the sarcolemmal membranes was increased. Periodic acid/Schiff staining showed that fibres with high lipid content also contained increased amounts of glycogen. There was no correlation, however, between lipid and glycogen storage and the intensity of the trichrome red staining. There was a slight predominance of type I adenosine triphosphatase reactions but in other respects a normal checkerboard pattern of the distribution of fibre types was seen. There was a pronounced increase in the amount of reaction product within individual fibres when succinic dehydrogenase and NADHtetrazolium reductase were used to stain for mitochondria (Fig. 2a).

Electronmicroscopy showed a considerable disturbance of the contractile apparatus with dilatation of intermyofibrillar spaces, splitting of myofibrils, and loss of the concerted arrangement of sarcomeres in many muscle fibres. More serious, however, was the great increase in aberrant mitochondria. Many of the intermyofibrillar and subsarcolemmal mitochondria did not have cristae of normal appearance and arrangement. Also they often contained cristallike inclusions and osmiophilic deposits of variable size and density (Fig. 2b and c). Quantitative morphometric stereological studies ${ }^{3}$ (ratio(SE)) confirmed that there was a distinct increase in the volume density (volume fraction occupied by the component) of the mitochondrial compartment (8.46, normal $4.28(1.84))$ and of the lipid (1.79, normal $0.79(0.50))$ and glycogen (3.11, normal 1.19 $(0 \cdot 87))$ deposits when compared with results in untrained age matched controls. Enzymehistochemistry showed normal activity of cytochrome oxidase (Fig. 2d). Unfortunately, no skeletal muscle from patient 2 was available for examination.

\section{The heart (patient 2)}

The myocardial fibres varied in size and shape and some were in considerable disarray (Fig. 3a) (type IIA and IIB by Maron and Roberts's classification ${ }^{4}$ ). Many of them contained vacuoles and showed myofibrillolysis. There were multiple scattered small scars in the myocardium of the left ventricle (Fig. $3 b)$, moreover a broad laminal fibrosis was found in the middle third of the septum (Fig. 3c), while the portions of the septum adjacent the right and left ventricle were spared. A small deparaffinised myo- 
cardial specimen was postfixed in glutaraldehyde and examined by electron microscopy. Despite artefacts due to paraffin embedding, myofibrils were clearly recognisable. There was a great increase in mitochondria throughout the myocardial fibres. The size of the mitochondria varied greatly, many contained large osmiophilic inclusions, apparently identical to those seen in the skeletal muscle mitochondria from patient 1 .

\section{Biochemical examination}

Rates of pyruvate and malate oxidation in the muscle homogenate were assayed by the method of Bookelman $e t a l^{5}$ with the glucose hexokinase system being replaced by $20 \mathrm{mmol} / 1$ creatine. $\mathbf{P}^{1}, \mathbf{P}^{5}$-di(adenosine-5'-) pentaphosphate (Ap5A) was added to inhibit adenylate kinase activity. ${ }^{6}$ Adenosine triphosphate production rates were determined in the presence of Ap5A and corrected for arsenite insensitivity. ${ }^{6}$ Cytochromes were assayed by the method of Bookelman et al. ${ }^{7}$ Carnitine was estimated by the method of Parvin and Pande. ${ }^{8}$

Biochemical examination did not show a clear cut defect in the energy producing pathway of the mitochondria. The cytochromes were present in normal amounts. Concentrations of total and non-esterified carnitine were normal in serum and muscle homogenates.

\section{Discussion}

Our patients and those described before ${ }^{1}$ probably had a hereditary disease of heart and skeletal muscle associated with congenital cataracts. On the basis of pathophysiological criteria the cardiomyopathy can be classified as a hypertrophic cardiomyopathy. In the patient who died the gross pattern of the heart was characteristic of this type of cardiomyopathy. The broad scar in the septum, however, was an unusual finding. It can only be supposed that it developed because of a concurrent disturbance of coronary perfusion in the severely hypertrophic myocardium.

Pronounced changes in the ultrastructure and distribution of mitochondria in the myocardial and skeletal muscle fibres were the most prominent histopathological findings. There are several reports of mitochondrial abnormalities in the skeletal muscles of patients with a heart disease..$^{9-11}$

A functional abnormality is likely in patient 1 because there was abnormally high lactic acidosis after exercise as a consequence of impaired pyruvate oxidation. Biochemical examination of muscle tissue, however, excluded the well known causes of disordered pyruvate oxidation. A disturbance of oxygen delivery to the mitochondria might explain the disordered mitochondrial function. Unfortunately we did not test for oxygen availability in vivo. Impaired oxygen delivery might also explain the occurrence of congenital cataract since the lens is vascularised for only a limited time during fetal development. Impaired oxygen delivery can cause cataract in experimental studies. ${ }^{12}$

Although we know of several patients with this syndrome in The Netherlands, no other cases have been described in published reports. We know of a few more Swiss patients with the syndrome (B Steinman, personal communication). The frequency of such cases is probably greater than the small number of reported cases suggests.

\section{References}

1 Sengers RCA, Ter Haar BGA, Trijbels JMF, Willems JH, Daniels O, Stadhouders AM. Congenital cataract and mitochondrial myopathy of skeletal and heart muscle associated with lactic acidosis after exercise. $\mathcal{F}$ Pediatr 1975; 86: 873-80.

2 McKusick VA. Mendelian inheritance in man. 6th ed. Baltimore: John Hopkins University Press, 1983.

3 Weibel ER. Stereological methods. Vol. 1: Practical methods for biological morphometry. London: Academic Press, 1979.

4 Maron BJ, Roberts WC. Quantitative analysis of cardiac muscle cell disorganization in the ventricular septum of patients with hypertrophic cardiomyopathy. Circulation 1979; 59: 689-706.

5 Bookelman H, Trijbels JMF, Sengers RCA, Janssen AJM, Veerkamp JH, Stadhouders AM. Pyruvate oxidation in rat and human skeletal muscle mitochondria. Biochem Med 1978; 20: 395-403.

6 Ruitenbeek W, Janssen AJM, Fischer JC, Sengers RCA, Trijbels JMF, Stadhouders AM. Investigation of the energy metabolism in diseased human muscular tissue. In: Scarlato G, Cerri C, eds. Mitochondrial pathology in muscle diseases. Padua: Piccin Nuova Libraria, 1983: 197-201.

7 Bookelman H, Trijbels JMF, Sengers RCA, Janssen AJM. Measurement of cytochromes in human skeletal muscle mitochondria, isolated from fresh and frozen stored muscle specimens. Biochem Med 1978; 19: 366-73.

8 Parvin R, Pande SV. Microdetermination of (-) carnitine and carnitine acetyltransferase activity. Anal Biochem 1977; 79: 190-201.

9 Bogousslavsky J, Perentes E, Deruaz JP, Regli F. Mitochondrial myopathy and cardiomyopathy with neurodegenerative features and multiple brain infarcts. $f \mathrm{Neurol} \mathrm{Sci}$ 1982; 55: 351-7.

10 Hübner G, Grantzow R. Mitochondrial cardiomyopathy with involvement of skeletal muscles. Virchows Arch [A] 1983; 399: 115-25.

11 Mastaglia FL, Thompson PL, Papadimitriou JM. Mitochondrial myopathy with cardiomyopathy, lactic acidosis and response to prednisone and thiamine. Aust NZ F Med 1980; 10: 660-4.

12 Varma SD, Chand D, Sharma YR, Kuck JF Jr, Richards RD. Oxidative stress on lens and cataract formation: role of light and oxygen. Curr Eye Res 1984; 3: 35-57. 